Journal of Mathematics and Statistics 2 (2): 391-394, 2006

ISSN 1549-3644

(C) 2006 Science Publications

\title{
Least Square Approximation by Linear Combination of Exponential Functions
}

\author{
Bahman Mehri, Dariush Shadman and Sadegh Jokar \\ Department of Mathematical Sciences, \\ Sharif University of Technology, Tehran, Iran
}

\begin{abstract}
Here we were concerned with least square approximation by exponential functions for given data. In this manuscript, we approximate the given data such that this approximant satisfies a differential equation. The case of nonlinear differential equations was also considered.
\end{abstract}

Key words: Exponential functions, Least Square Approximation,Linear Differential Equation

\section{INTRODUCTION}

A continuous function $f$ is defined on some interval $I$ for which a set of data points is given. We would like to find a curve which fits the data in the sense that it is the best approximation to the function $f$ in $\ell_{2}$ norm $^{[2]}$. The approximation is normally made using a polynomial of certain degree, Chebyshev polynomials, etc.

Here we propose to approximate the function $f$ at the given set of points using exponential functions which are the solution of a linear differential equation with constant coefficients. This approximant gives the least square approximation of the solution of a linear second order differential equation(Section 2). Later this method is extended to the case of higher order differential equations(Section 3). The case of nonlinear differential equations is also considered(Section 4).

Definition 1: An $n$-dimensional vector space $U$ of functions on a domain $X$ is said to be Haar space if the only element $U$ of which has more than $n-1$ roots in $X$ is the zero element.

Theorem $1^{[1]}$ : Let $U$ have the basis $\left\{u_{1}, \ldots, u_{n}\right\}$. The following properties are equivalent:

- $\quad U$ is a Haar space.

- $\operatorname{det}\left(u_{i}\left(x_{j}\right)\right) \neq 0$ for any set of distinct points $x_{1}, \ldots, x_{n}$ in $X$.

From this theorem, it can be easily seen that if $\left\{\lambda_{i}\right\}_{i=1}^{N}$ are distinct then the set of exponential functions $\left\{e^{\lambda_{1} x}, e^{\lambda_{2} x}, \ldots, e^{\lambda_{N} x}\right\}$ form a Haar subspace and therefore we can use it for least square approximation.

\section{Least Square Approximation by Exponential Functions for Second order ODE}

Suppose we are given $\mathrm{n}$ pairs of data $\left(x_{1}, y_{1}\right), \ldots,\left(x_{n}, y_{n}\right)$. The problem is to find $\lambda_{1}, \lambda_{2}$ such that the function $c_{1} e^{\lambda_{1} x}+c_{2} e^{\lambda_{2} x}$ is the best approximation in $\ell_{2}$ norm for given data and it is a solution of the differential equation of the second order. First we consider the following theorem.

Theorem 2: Suppose the nodes $\{a+i h\}_{i=0}^{4}$ are given for some $h>0$. If

$$
y^{\prime \prime}+A y^{\prime}+B y=0
$$

then

$$
\begin{aligned}
& \mathrm{y}(\mathrm{a})-2 \mathrm{y}(\mathrm{a}+2 \mathrm{~h})+\mathrm{y}(\mathrm{a}+4 \mathrm{~h})+ \\
& \mathrm{A}\left(\int_{\mathrm{a}+2 \mathrm{~h}}^{\mathrm{a}} y(t) d t+\int_{\mathrm{a}+2 \mathrm{~h}}^{\mathrm{a}+4 \mathrm{~h}} y(t) d t\right)+ \\
& B\left(\int_{\mathrm{a}+2 \mathrm{~h}}^{\mathrm{a}}(a-t) y(t) d t+\int_{\mathrm{a}+2 \mathrm{~h}}^{\mathrm{a}+4 \mathrm{~h}}(a+4 h-t) y(t) d t\right)=0 \\
& \text { Proof: First integrate on }[a+2 h, x]:
\end{aligned}
$$

$$
\begin{aligned}
& \int_{\mathrm{a}+2 \mathrm{~h}}^{\mathrm{x}}\left[y^{\prime \prime}(t)+A y^{\prime}(t)+B y(t)\right] d t=y^{\prime}(x)-y^{\prime}(a+2 h) \\
& +A(y(x)-y(a+2 h))+B \int_{\mathrm{a}+2 \mathrm{~h}}^{\mathrm{x}} y(t) d t=0
\end{aligned}
$$

Integrating this equation once more to get:

$\int_{\mathrm{a}+2 \mathrm{~h}}^{\mathrm{x}} y^{\prime}(t) d t-y^{\prime}(a+2 h)(x-(a+2 h))+A \int_{\mathrm{a}+2 \mathrm{~h}}^{\mathrm{x}} y(t) d t$

$-A y(a+2 h)(x-(a+2 h))+B \int_{\mathrm{a}+2 \mathrm{~h}}^{\mathrm{x}} y^{\prime}(t) d t=0$

and finally the following formula is obtained.

$y(x)-y(a-2 h)-y^{\prime}(a+2 h)(x-(a+2 h))+$

$A \int_{a+2 h}^{x} y(t) d t-A y(a+2 h)(x-(a+2 h))+$

$B \int_{a+2 h}^{x}(x-t) y(t) d t=0$

Now setting $x=a$ and $x=a+4 h$ in $\operatorname{Eq}(2.3)$ we get 


$$
\begin{aligned}
& y(a)-y(a+2 h)-y^{\prime}(a+2 h)(a-(a+2 h))+ \\
& A \int_{a+2 h}^{a} y(t) d t-A y(a+2 h)(a-(a+2 h))+ \\
& B \int_{a+2 h}^{a}(a-t) y(t) d t=0 \\
& \text { and, } \\
& y(a+4 h)-y(a+2 h)- \\
& y^{\prime}(a+2 h)(a+4 h-(a+2 h))+ \\
& A \int_{a+2 h}^{a+4 h} y(t) d t-A y(a+2 h)(a+4 h-(a+2 h))+ \\
& B \int_{a+2 h}^{a+4 h}(a+4 h-t) y(t) d t=0
\end{aligned}
$$

Adding Eqs(2.4) and (2.5) Eq(2.2) is obtained. Example 2.2. Suppose the pairs of nodes $x_{i}=\frac{i}{8}, 0 \leq i \leq 8$ and the values $y_{i}=e^{x_{i}}+e^{2 x_{i}}, 0 \leq i \leq 8$ are given. The approximate solution with Simpson rule is:

$$
y_{\text {app }}=1.0058 e^{1.9977 x}+0.99426 e^{0.99617 x}
$$

where the exact solution is $y_{\text {exact }}=e^{2 x}+e^{x}$.

Figure 1 shows the approximate and the exact solution of this example.

Example 2.3. Here the pairs of nodes

$x_{i}=\frac{i}{8}, 0 \leq i \leq 8$ and the values

$y_{i}=3 e^{-x_{i}}+e^{x_{i}}, 0 \leq i \leq 8$ are given. The approximate solution by Simpson rule is

$$
y_{\text {app }}=2.9994 e^{-1.0003 x}+1.0006 e^{0.99964 x}
$$

and the exact solution is $y_{\text {exact }}=3 e^{-x_{i}}+e^{x_{i}}$.

Figure 2 shows the approximate and the exact solutions of this example.

\section{Least square approximation by exponential functions for higher order ODE's}

Here the problem in the previous section is generalized. Suppose we are given $\mathrm{n}$ vectors of data

$$
\begin{aligned}
& \left(x_{1}, y_{1}, y_{1}^{\prime}, \ldots, y_{1}^{(m-2)}\right),\left(x_{2}, y_{2}, y_{2}^{\prime}, \ldots, y_{2}^{(m-2)}\right), \\
& \ldots,\left(x_{n}, y_{n}, y_{n}^{\prime}, \ldots, y_{n}^{(m-2)}\right)
\end{aligned}
$$

The problem is to find a differential equation of order $m$ with constant coefficients such that the solution of this differential equation is the least square approximation of given data. Therefore we should find $\lambda_{1}, \lambda_{2}, \ldots, \lambda_{m}$ such that the function $\sum_{k=1}^{m} c_{k} e^{\lambda_{k} x}$ is the best approximation in $\ell^{2}$ norm and it is a solution of some differential equation of order $\mathrm{m}$. The following theorem is a generalization of theorem 2 .
Theprem 3.1. Suppose $a, a+h, \ldots, a+4 m h$ is given for some $h>0$. Form

$$
\begin{aligned}
& y^{(m)}+A_{m-1} y^{(m-1)}+A_{m-2} y^{(m-2)}+ \\
& \ldots+A_{1} y^{\prime}+A_{0} y=0
\end{aligned}
$$

we have

$$
\begin{aligned}
& y^{(m-2)}(a)-2 y^{(m-2)}(a+2 h)+y^{(m-2)}(a+4 h)+ \\
& A_{m-1}\left(y^{(m-3)}(a)-2 y^{(m-3)}(a+2 h)+y^{(m-2)}(a+4 h)\right)+ \\
& \ldots+A_{1}\left(\int_{a+2 h}^{a} y(t) d t+\int_{a+2 h}^{a+4 h} y(t) d t\right)+ \\
& A_{0}\left(\int_{a+2 h}^{a}(a-t) y(t) d t+\int_{a+2 h}^{a+4 h}(a+4 h-t) y(t) d t\right)=0
\end{aligned}
$$

Proof. The proof is similar to Theorem (2.1).

Example 3.2. Suppose we are given the pairs of nods

$x_{i}=\frac{i}{12}, 0 \leq i \leq 12$ and the values

$y_{i}=e^{-x_{i}}+e^{2 x_{i}}-e^{3 x_{i}}, 0 \leq i \leq 12$. The

approximate solution with Simpson rule is:

$$
\begin{aligned}
y_{\text {app }}= & -1.0084 e^{2.9973 x}+1.0068 e^{2.0084 x} \\
& +1.0016 e^{-0.9978 x}
\end{aligned}
$$

where the exact solution is $y_{\text {exact }}=e^{-x}+e^{2 x}-e^{3 x}$.

Figure 3 shows the exact and approximate solution of this example.

\section{Least square approximation by exponential function}

\section{for nonlinear ODE's}

Here we would like to find an approximate solution for nonlinear ODE's by the method of the previous section. One way to solve this problem is to approximate the unknown function $y$ by linear combination of exponential functions, i.e. such that $y(x) \simeq s(x)=\sum_{k=1}^{n} c_{k} e^{\lambda_{k} x}$. Then the nonlinear least square approximation is used such that $\sum_{i=1}^{n}\left|s^{\prime}\left(x_{i}\right)-f\left(x_{i}, s\left(x_{i}\right)\right)\right|^{2}$ is minimized.

However the obove nonlinear approximation method is very difficult to compute. Instead we introduce the following aproach. First we look at the first order equation $y^{\prime}=f(x, y)$. To begin we use Runge Kutta method of foruth order to find an approximate solution at some points. Then we use these data to find a linear ODE such that the solution of this ODE is an approximate solution of the nonlinear ODE.

Example 4.1. The approximate solution of the following nonlinear differential equation

$$
\left\{\begin{array}{c}
y^{\prime}=\sin (y)+\cos (x), x \in\left[0, \frac{\pi}{2}\right] \\
y(0)=0
\end{array}\right.
$$

is given in Figure 4. 
Example 4.2. Suppose it is required to solve the following nonlinear differential equation

$$
\left\{\begin{array}{c}
y^{\prime \prime}+\sin (y)=0, x \in[0,10] \\
y(0)=\frac{\pi}{2}, y^{\prime}(0)=0
\end{array}\right.
$$

Figure 5 shows the approximate solution.

Example 4.3. Next the following nonlinear differential equation is considered.

$$
\left\{\begin{array}{c}
y^{\prime \prime}+0.2\left(1-y^{2}\right) y^{\prime}+y=0, x \in[0,10] \\
y(0)=1, y^{\prime}(0)=0
\end{array}\right.
$$

Figure 6 shows the approximate solution.

\section{CONCLUSION}

In this manuscript we deal with the problem of finding a function which is a linear combination of exponential functiona and is the least square approximation of the given data. Finally we approximate the nonlinear differential equation by linear differential equation with constant coefficients in a finite domain. We can also generalize this method for the solution of PDEs.
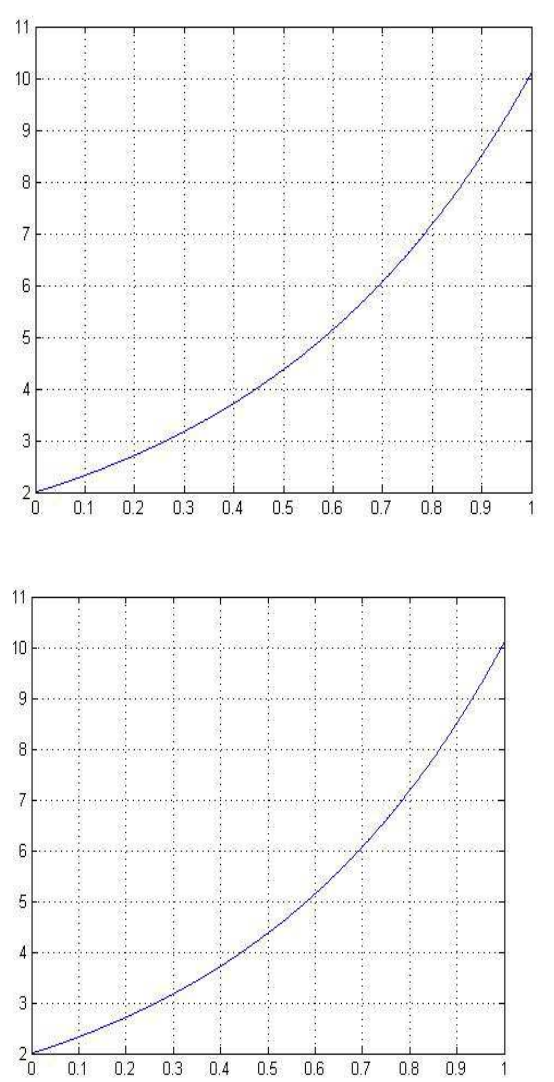

Fig. 1: Approximate and Exact solution for Example 2.2.
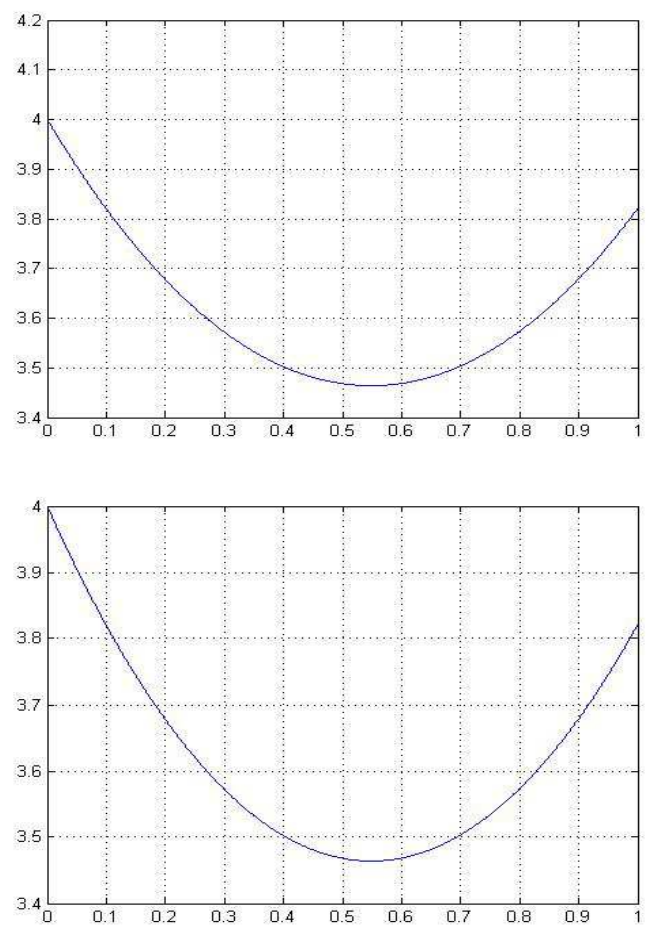

Fig. 2: Approximate and Exact solution for Example 2.3.
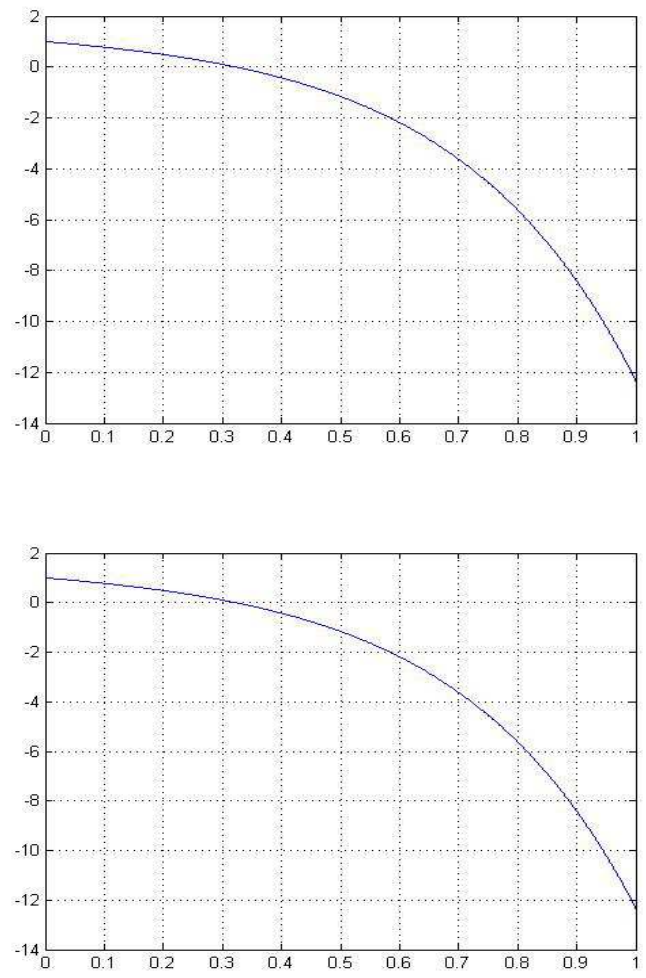

Fig. 3: Approximate and Exact solution for Example 3.2 . 


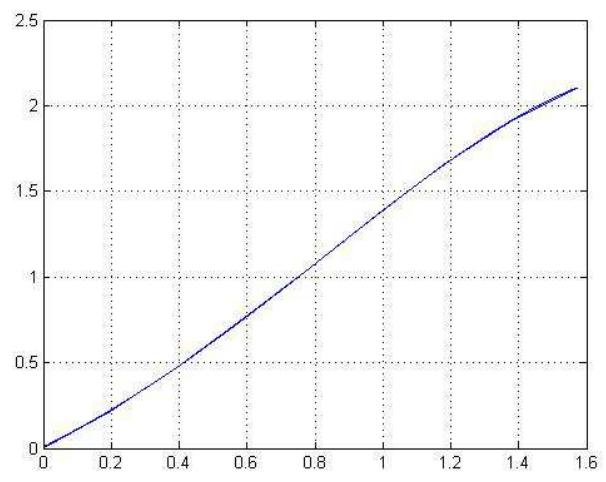

Fig. 4: Approximate solution for Example 4.1.

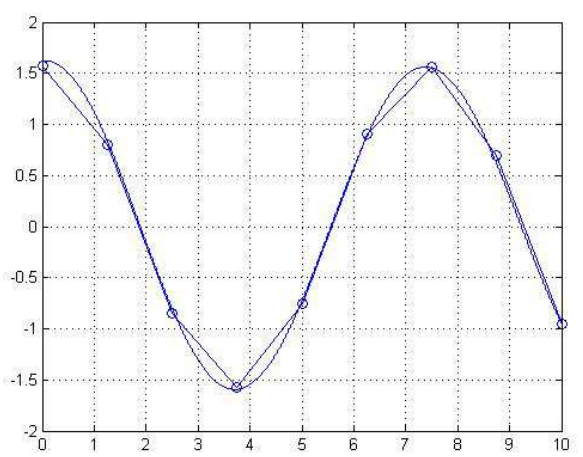

Fig. 5: Approximate solution for Example 4.2.

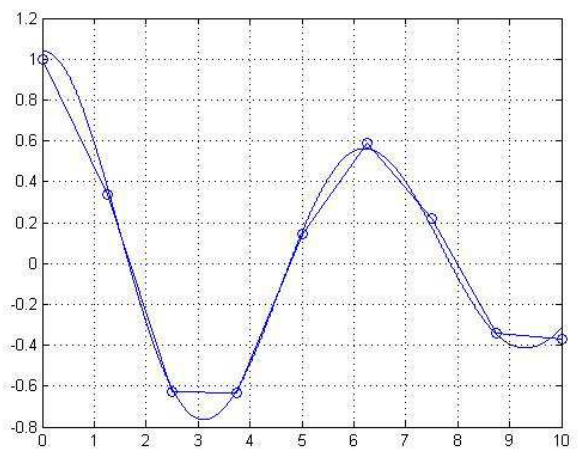

Fig. 6: Approximate solution for Example 4.3.

\section{ACKNOWLEDGMENT}

This work is supported by Sharif University of Technology.

\section{REFERENCES}

1. Cheney, E. W. and W. A. Light, A Course in Approximation Theory, Brooks/Cole, Pacific Grove, CA, 2000.

2. T.J. Rivlin, An Introduction to the Approximation of Functions, Dover, New York, 1981. 